\title{
Identification of altered pathways in breast cancer based on individualized pathway aberrance score
}

\author{
SHENG-HONG SHI, WEI ZHANG, JING JIANG and LONG SUN \\ Department of Breast Surgery, Ningbo No. 2 Hospital, Ningbo, Zhejiang 315000, P.R. China
}

Received July 30, 2015; Accepted December 20, 2016

DOI: $10.3892 / 01.2017 .6292$

\begin{abstract}
The objective of the present study was to identify altered pathways in breast cancer based on the individualized pathway aberrance score (iPAS) method combined with the normal reference (nRef). There were 4 steps to identify altered pathways using the iPAS method: Data preprocessing conducted by the robust multi-array average (RMA) algorithm; gene-level statistics based on average $Z$; pathway-level statistics according to iPAS; and a significance test dependent on 1 sample Wilcoxon test. The altered pathways were validated by calculating the changed percentage of each pathway in tumor samples and comparing them with pathways from differentially expressed genes (DEGs). A total of 688 altered pathways with $\mathrm{P}<0.01$ were identified, including kinesin (KIF)- and polo-like kinase (PLK)-mediated events. When the percentage of change reached $50 \%, 310$ pathways were involved in the total 688 altered pathways, which may validate the present results. In addition, there were 324 DEGs and 155 common genes between DEGs and pathway genes. DEGs and common genes were enriched in the same 9 significant terms, which also were members of altered pathways. The iPAS method was suitable for identifying altered pathways in breast cancer. Altered pathways (such as KIF and PLK mediated events) were important for understanding breast cancer mechanisms and for the future application of customized therapeutic decisions.
\end{abstract}

\section{Introduction}

Breast cancer is characterized by a distinct metastatic pattern involving the regional lymph nodes, bone marrow, lungs and the liver (1). It is the most common type of cancer diagnosed among women and the second leading cause of cancer mortality among women following lung cancer (2). A family history of

Correspondence to: Dr Sheng-Hong Shi, Department of Breast Surgery, Ningbo No. 2 Hospital, 41 Northwest Street, Ningbo, Zhejiang 315000, P.R. China

E-mail: shenghongshi@yeah.net

Key words: breast cancer, average Z, individualized pathway aberrance score, altered pathways breast cancer and several other factors (including female sex, old age and exposure to ionizing radiation) increase the risk of developing breast cancer (3). In addition, 5-10\% of breast cancer cases are caused by inherited gene mutations (4). Several gene markers have been identified to predict responses to therapeutic regimens, such as receptor tyrosine-protein kinase erbB-2 and Stearoyl-CoA desaturase-1 (5-7). However, development remains necessary to understanding the mechanisms of breast cancer, in order to customize anticancer therapies and to identify altered pathways in an individual with breast cancer.

Pathway analysis has become the first choice for gaining insight into the underlying biology of genes and proteins, as it reduces complexity and has increased explanatory power (8). Existing pathway analysis techniques are predominantly focused on discovering altered pathways between normal and cancer groups and are not suitable for identifying the pathway aberrance that may occur in an individual sample (9). A simple way to identify an individual's pathway aberrance is to compare normal and tumor data from the same individual. However, matched normal data from the same individual is often unavailable in clinical situations. Therefore, the present study applied a new approach for the personalized identification of altered pathways, making special use of accumulated normal data in cases when a patient's matched normal data were unavailable (10).

The present study identified altered pathways in breast cancer based on the individualized pathway aberrance score (iPAS) method which included data preprocessing, gene-level statistics, pathway-level statistics and a significant test. The altered pathways were validated by comparison with pathways based on differentially expressed genes (DEGs), and by calculating the percentage of changed pathways in breast cancer samples.

\section{Materials and methods}

Gene expression data. In the present study, the gene expression profile with accession number E-GEOD-10780 (11) was recruited from the ArrayExpress database (http://www.ebi .ac.uk/arrayexpress/). E-GEOD-10780, which was presented on the A-AFFY-44-Affymetrix GeneChip Human Genome U133 Plus 2.0 (Affymetrix, Inc., Santa Clara, CA, USA), comprised of 143 normal control samples and 42 breast cancer samples. The gene expression profile on probe level was 
converted into gene symbol level. Subsequent to removing the duplicated symbols, a total of 20,102 gene symbols were obtained for additional analysis.

Pathway data. Information from gene sets representing biological pathways was downloaded from the reactome pathway database (http://www.reactome.org/) (12). Reactome is an online curated resource for human pathway data and provides infrastructure for computation across the biological reaction network (12). Pathways involving a small number of genes are easily understood by researchers. Therefore, for the present study pathways with a gene set of $>100$ were filtered out. In addition, the present study also eliminated pathways that had a null set with the gene expression profile E-GEOD-10780. Finally, 1,013 pathways were identified, which consisted of 5,182 genes.

Individualized analysis for pathways. To identify altered pathways in an individual breast cancer sample, the iPAS method was employed (10), which includes 4 steps: Data preprocessing (Fig. 1A); gene-level statistics (Fig. 1B); pathway-level statistics (Fig. 1C); and a significant test (Fig. 1D).

Data pre-processing. Prior to analysis, a standard pre-treatment was conducted to control the quality of the gene expression profiles. For normal genes, background corrections and normalization were carried out using the robust multi-array average (RMA) algorithm and the quantile based algorithm to eliminate the influence of nonspecific hybridization $(13,14)$. The Micro Array Suite 5.0 (MAS 5.0) algorithm was then applied to revise perfect match and mismatch value (15), and median polish method was applied to summarize the expression value (13). All normal control samples were regarded as references (nRef) in the present study. For individual breast cancer cases, the present study performed uniformly standardized normalization following the combination of the single tumor microarray with all nRef samples.

Gene-level statistics. Standardizing the gene expression on the gene-level via mean and standard deviation (SD) from datasets is often used in microarray analysis. In the present study, the individual tumor sample gene expression level was standardized based on the mean and SD of the normal references. This formula was defined as:

$$
Z_{i j}=\frac{T_{i j}-\operatorname{mean}\left(N_{j}\right)}{\operatorname{stdev}\left(N_{j}\right)}
$$

Where mean $\left(\mathrm{N}_{\mathrm{j}}\right)$ symbolized the mean expression value of the genes of the nRef, stdev $\left(\mathrm{N}_{\mathrm{j}}\right)$ symbolized the SD of the normal, $\mathrm{T}_{\mathrm{ij}}$ symbolized the expression value of $\mathrm{i}$-th tumor gene and $Z_{i j}$ symbolized the standardized expression value of $i$-th tumor gene, where the number of genes belonging to the gene was i.

Pathway-level statistics based on average $Z$. The average $Z$ method was selected to evaluate iPAS by utilizing the nRef. A vector $Z=\left(z_{n}\right)$ denoted the expression status of a pathway, where $z_{i}$ symbolizes the standardized expression value of the $i$-th gene and is derived from mean and SD of the nRef. $n$ was the number of genes belonging to the pathway. The iPAS was calculated as following:

$$
\mathrm{iPAS}=\sum_{\mathrm{j}}^{\mathrm{n}} Z_{\mathrm{i}}
$$

Significant measurement. A one sample Wilcoxon-test was conducted for normal and tumor pathway statistics values to estimate the significance of the pathways (16). All collected normal samples for the nRef were sequentially compared with the nRef to yield statistics of the null distribution. A P-value was produced according to comparison between this null distribution and a statistic from a single tumor case, and was adjusted by false discovery rate (FDR). A pathway with $\mathrm{P}<0.01$ was considered as altered pathway compared with nRef.

Hierarchical clustering analysis of altered pathways. To assess the classification performance of altered pathways, a hierarchical clustering analysis was applied across 42 tumor samples and 143 normal control samples using the Gene Cluster 3.0 (Human Genome Center, University of Tokyo, Tokyo, Japan) program. The clustering algorithm was set to complete linkage clustering using an uncentered correlation. Ideally, the samples should be classified into 2 major clusters: Tumor cases and normal controls. The present study tested the method by measuring the percentage of test samples that could be correctly classified. Accuracy is the fraction of correctly classified samples over all samples (17).

$$
\text { Accuracy }=\frac{\mathrm{TN}+\mathrm{TP}}{\mathrm{TN}+\mathrm{TP}+\mathrm{FN}+\mathrm{FP}}
$$

TP (true positive) represents the number of positive samples correctly predicted as positive, TN (true negative) represents the number of negative samples correctly predicted as negative, FP (false positive) represents the number of negative samples incorrectly predicted as positive and FN (false negative) represents the number of positive samples incorrectly predicted as negative.

Validation of the altered pathways. The present study applied 2 methods to validate altered pathways obtained from individualized analysis using the nRef, one was comparing with the traditional approach according to DEGs to identify pathways, and the other was by calculating the percentage of each changed pathway in tumor samples.

DEGs based pathway analysis. The linear models for microarray data (Limma) package (18) was utilized to explore DEGs between the patients with breast cancer and the normal controls. Only the genes with FDR adjusted P-values of $<0.01$ and log fold change of $>2$ were classed as DEGs. The Kyoto Encyclopedia of Genes and Genomes (KEGG) pathway enrichment analysis for DEGs was conducted based on the Database for Annotation, Visualization and Integrated Discovery (19), which implemented the expression analysis systematic explorer test to selected pathways with the criterion $\mathrm{P}<0.01(20)$.

Pathway changed percent. To validate altered pathways in tumors which were identified by iPAS method combined with nRef, the present study counted the percentage change for 

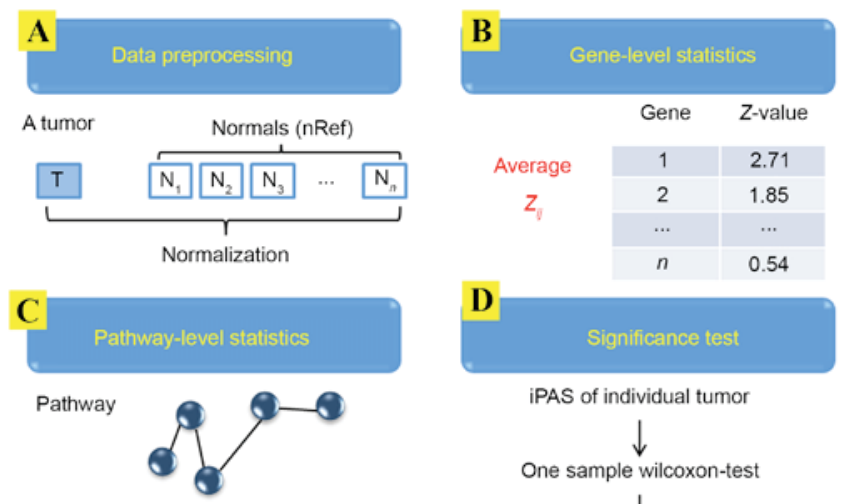

Individualized pathway aberrance score (iPAS)

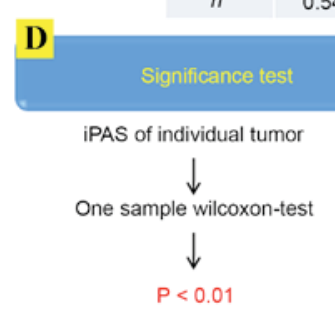

Figure 1. Schematic flow showing the 4 stages of individualized pathway analysis based on the iPAS method using normal reference. iPAS, individualized pathway aberrance score. (A) Data preprocessing schematic flow. (B) Gene-level statistical analysis. (C) Pathway-level statistical analysis. (D) Statistical significance test.

each pathway in breast cancer samples. Firstly, by taking the distribution character of each pathway statistic value in normal and tumor samples, the empirical P-value of each pathway in a tumor individual compared with nRef was detected. The amount of P-values $<0.01$ were then statistically counted in order to obtain the changed percentage for each pathway in all breast cancer cases.

Statistical analysis. In the present study, the one sample Wilcoxon test (using SPSS v.19.0; IBM SPSS, Armonk, NY, USA) was utilized to estimate the significance of the pathways, of which $\mathrm{P}$ values also were calculated. $\mathrm{P}<0.05$ was considered to indicate a statistically significant difference. However, to be confident in the validity of these results, $\mathrm{P}<0.01$ was the statistically significant threshold in the current study.

\section{Results}

Identification of altered pathways. In the present study, 143 normal control samples in the gene expression profile E-GEOD-10780 were defined as nRef of 42 tumor samples. The present study performed quantile normalization for tumor genes to evaluate their gene-level statistics. A total of 1,013 pathways were identified from the reactome pathway database. The present study extracted gene-level statistic values of all genes enriched in one pathway, and denoted the mean value to pathway-level statistics of this pathway. With a threshold value of $\mathrm{P}<0.01$, a total of 688 altered pathways were explored for breast cancer. The cluster analysis of using Average $\mathrm{Z}$ as the iPAS method based on individual breast cancer and normal controls was also shown in Fig. 2. As presented in Fig. 2, $\mathrm{TP}=31, \mathrm{FP}=11, \mathrm{TN}=143$, while $\mathrm{FN}=0$, thus the accuracy of classification equaled to $94.05 \%$, which indicated that the samples possessed good classification.

In addition, the top $5 \%$ of the 688 altered pathways are shown in Table I. Polo-like kinase (PLK) -mediated events, phosphorylation of proteins involved in $\mathrm{G}_{1} / \mathrm{S}$ transition by active cyclin $\mathrm{E} / \mathrm{Cdk} 2$ complexes, $\mathrm{G}_{2} / \mathrm{M}$ DNA replication checkpoint, FGFR2b ligand binding and activation and cyclin

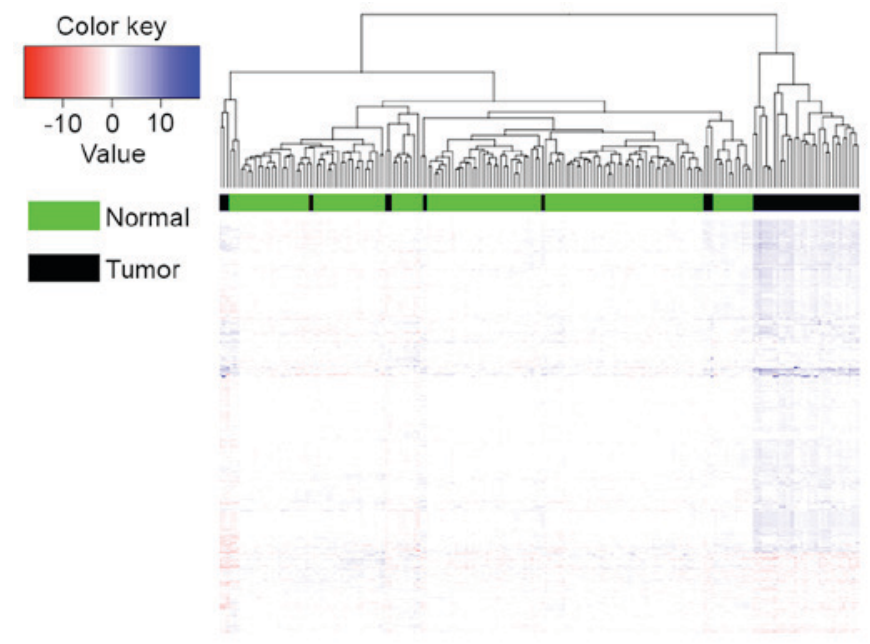

Figure 2. Cluster of using Average $Z$ as the iPAS method based on individual breast cancer and normal controls. iPAS, individualized pathway aberrance score.

A/B1 associated events during $\mathrm{G}_{2} / \mathrm{M}$ transition were the most significant pathways with a P-value of 4.38E-18.

Gene compositions of altered pathways. Pathways involve several genes, which work together to perform one biological process or to regulate certain biological functions. To additionally identify the functions and properties of altered pathways, the present study investigated their gene compositions at the gene expression level. The PLK-mediated events pathway was comprised of 13 genes (centromere protein F, CENPF; E1A binding protein p300, EP300; forkhead box protein M1, FOXM1; MYB proto-oncogene like 2, MYBL2; polo-like kinase 1, PLK1; lin-37 DREAM MuvB complex component, LIN37; RB binding protein 4, chromatin remodeling factor, RBBP4; WEE $1 \mathrm{G} 2$ checkpoint kinase, WEE1; cyclin B1, $C C N B 1$; protein kinase, membrane associated tyrosine/threonine 1, PKMYT1; cyclin B2, CCNB2; cell division cycle 25A, $C D C 25 A$ and cell division cycle 25C CDC 25C), and Fig. 3 illustrates expression patterns of genes in this pathway across normal and breast cancer samples. The present study identified that the gene-level statistic in tumors was disturbed relative to that in normal samples, and in normal samples the gene-levels for the 13 genes were similar in general. Therefore, it may be inferred that different gene-levels lead to the production of altered pathways in breast cancer compared with nRef.

Comparison with pathways based on DEGs. The present study identified a total of 324 DEGs between breast cancer and normal controls with thresholds of $\mathrm{P}<0.01$ and log fold change of $>2$. Taking the intersection with 5,182 genes contained in 1,013 pathways, only 155 common genes were detected.

Results of the KEGG pathway enrichment analysis showed that 324 DEGs were enriched in 9 significant pathways under the condition of $\mathrm{P}<0.01$ (Table II). The most significant pathways were focal adhesion $(\mathrm{P}=4.01 \mathrm{E}-05), \mathrm{ECM}$-receptor interaction $(\mathrm{P}=4.73 \mathrm{E}-05)$ and cytokine-cytokine receptor interaction $(\mathrm{P}=2.90 \mathrm{E}-04)$. When performing KEGG enrichment analysis for common genes, notably, the 155 common genes also enriched in the same 9 pathways, however the properties 
Table I. Top 5\% of 688 altered pathways with $\mathrm{P}<0.01$ in breast cancer.

Pathway

P-value

Polo-like kinase mediated events

4.38E-18

Phosphorylation of proteins involved in G1/S transition by active Cyclin E/Cdk2 complexes

4.38E-18

$\mathrm{G}_{2} / \mathrm{M}$ DNA replication checkpoint

$4.38 \mathrm{E}-18$

FGFR2b ligand binding and activation

$4.38 \mathrm{E}-18$

Cyclin A/B1 associated events during $\mathrm{G}_{2} / \mathrm{M}$ transition

4.38E-18

Deposition of new CENPA-containing nucleosomes at the centromere

$1.27 \mathrm{E}-17$

Nucleosome assembly

$1.27 \mathrm{E}-17$

Kinesins

$1.50 \mathrm{E}-17$

Removal of the flap intermediate from the C-strand

$3.47 \mathrm{E}-17$

$\mathrm{G}_{1} / \mathrm{S}$-specific transcription

4.99E-17

Phosphorylation of emi1

4.99E-17

Removal of the flap intermediate

$8.93 \mathrm{E}-17$

Chromosome maintenance

$8.93 \mathrm{E}-17$

$\mathrm{G}_{0}$ and early $\mathrm{G}_{1}$

$8.93 \mathrm{E}-17$

FGFR1b ligand binding and activation

$1.02 \mathrm{E}-16$

Cyclin B2 mediated events

$1.05 \mathrm{E}-16$

CHL1 interactions

$1.24 \mathrm{E}-16$

Phosphorylation of the APC/C

$1.87 \mathrm{E}-16$

Meiotic recombination

$1.87 \mathrm{E}-16$

RNA polymerase I promoter opening

$2.06 \mathrm{E}-16$

Notch-HLH transcription pathway

2.33E-16

Type I hemidesmosome assembly

3. $41 \mathrm{E}-16$

E2F mediated regulation of DNA replication

$3.41 \mathrm{E}-16$

Unwinding of DNA

$3.41 \mathrm{E}-16$

Telomere Maintenance

$4.00 \mathrm{E}-16$

Inactivation of $\mathrm{APC} / \mathrm{C}$ via direct inhibition of the $\mathrm{APC} / \mathrm{C}$ complex

$4.00 \mathrm{E}-16$

Inhibition of the proteolytic activity of $\mathrm{APC} / \mathrm{C}$ required for the onset

$4.00 \mathrm{E}-16$

of anaphase by mitotic spindle checkpoint components

$\mathrm{G}_{2} / \mathrm{M}$ checkpoints

$4.00 \mathrm{E}-16$

Mitotic spindle checkpoint

$4.66 \mathrm{E}-16$

DNA strand elongation

$4.66 \mathrm{E}-16$

Processive synthesis on the lagging strand

$4.66 \mathrm{E}-16$

E2F-enabled inhibition of pre-replication complex formation

$5.20 \mathrm{E}-16$

Telomere C-strand (lagging strand) synthesis

$5.80 \mathrm{E}-16$

Activation of the pre-replicative complex

$5.80 \mathrm{E}-16$

were different, such as P-value, count and enriched genes The most significant term of common genes was the peroxisome proliferator-activated receptor signaling pathway with $\mathrm{P}=3.44 \mathrm{E}-04$. In addition, cytokine-cytokine receptor interactions had the largest count of 18 , whilst the next was pathways in cancer with a count of 14 . Although the DEGs are not entirely included by 5,182 pathway genes, the 9 KEGG pathways were all involved in 688 altered pathways, which indicates that the present method was used to identify altered pathways.

Validation of altered pathways based on changed percent. The present study calculated percentage of changed pathways among 42 breast cancer samples, and listed the 47 pathways with changes in $>80 \%$ tumor samples (Table III). The 47 pathways were part of 688 altered pathways. Kinesins (KIFs) and
PLK mediated events were changed in 39 individuals (92.86\%), the next were chromosome maintenance, meiotic recombination, deposition of new centromere protein A-containing nucleosomes at the centromere, nucleosome assembly and $\mathrm{G}_{2} / \mathrm{M}$ (DNA damage) DNA replication checkpoint changed in 38 individuals $(90.48 \%)$. If the changed percentage was equal to $50 \%$, a total of 310 terms were obtained, which are also involved in 688 altered pathways. This may contribute to validation of the present results.

The gene composition of PLK mediated events had been analyzed (Fig. 3), and CCNB1 was the common gene. Its gene-level statistic value across different individual had clear differences. In KIFs, there were 21 genes, KIF20A, centromere protein E (CENPE), KIF2C, KIF3A, KIFAP3, KIF4A, rac GTPase activating protein 1 (RACGAP1), KIF2A, KIF3C, 
Table II. Kyoto encyclopedia of genes and genomes pathways with $\mathrm{P}<0.01$ based on DEGs and common genes.

\begin{tabular}{|c|c|c|c|c|}
\hline \multirow[b]{2}{*}{ Pathway } & \multicolumn{2}{|c|}{ P-value } & \multicolumn{2}{|c|}{ Count } \\
\hline & DEGs & Common & DEGs & Common \\
\hline Focal adhesion & $4.01 \mathrm{E}-05$ & $5.39 \mathrm{E}-03$ & 17 & 11 \\
\hline Extracellular matrix-receptor interaction & 4.37E-05 & $5.59 \mathrm{E}-03$ & 11 & 7 \\
\hline Cytokine-cytokine receptor interaction & $2.90 \mathrm{E}-04$ & $1.54 \mathrm{E}-03$ & 18 & 14 \\
\hline Peroxisome proliferator-activated receptor signaling pathway & $1.68 \mathrm{E}-03$ & $3.44 \mathrm{E}-04$ & 8 & 8 \\
\hline Pathways in cancer & $3.53 \mathrm{E}-03$ & $9.85 \mathrm{E}-03$ & 18 & 14 \\
\hline Adipocytokine signaling pathway & $6.68 \mathrm{E}-03$ & $9.41 \mathrm{E}-03$ & 7 & 6 \\
\hline Aldosterone-regulated sodium reabsorption & $7.39 \mathrm{E}-03$ & $7.88 \mathrm{E}-03$ & 5 & 5 \\
\hline Chemokine signaling pathway & 8.71E-03 & $9.51 \mathrm{E}-03$ & 11 & 10 \\
\hline Oocyte meiosis & $9.05 \mathrm{E}-03$ & $5.31 \mathrm{E}-03$ & 8 & 8 \\
\hline
\end{tabular}

DEGS, differentially expressed genes.

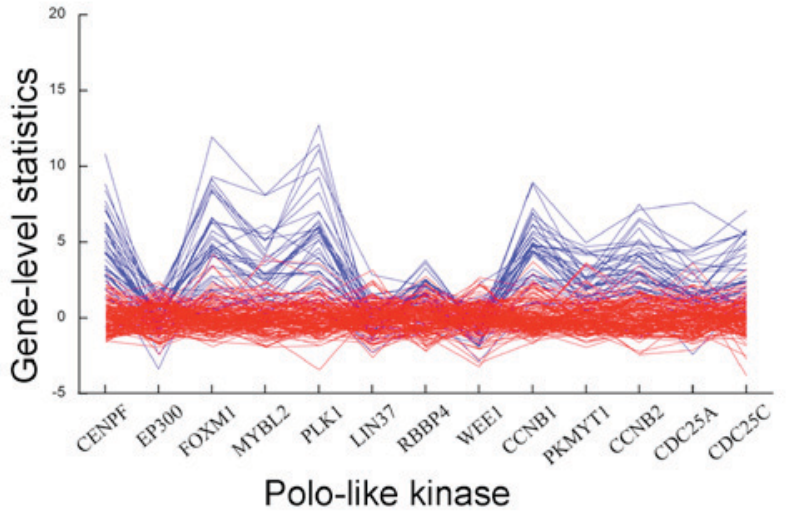

Figure 3. Gene level statistics in Polo-like kinase mediated events. Each line represents a sample. Blue, tumor; red, normal.

kinesin light chain 1 (KLC1), KIF5A, kinesin light chain 2 (KLC2), KIF5B, KIF5C, KIF11, KIFC1, KIF22, KIF15, KIF18A, KIF3B and KIF23, of which KIF20A and KIF11 were common genes. The standardized gene expression pattern for this pathway differed between tumor and normal (Fig. 4). A number of the genes deviated from the mean of the nRef and expression pattern of a gene varied markedly between tumor and normal samples. For an individual, different genes had their own gene-level statistics.

\section{Discussion}

The present study identified altered pathways in breast cancer using a new method (iPAS method combined with nRef), and validated its feasibility based on the percentage of changed pathways in breast cancer samples and comparison with KEGG pathways. KEGG pathways and pathways with a changed percentage of $>50 \%$ were parts of the altered pathways, which indicated that the iPAS method to identify altered pathways in breast cancer was feasible. The present results indicated that a total of 688 altered pathways were identified, such as KIF- and PLK-mediated events.

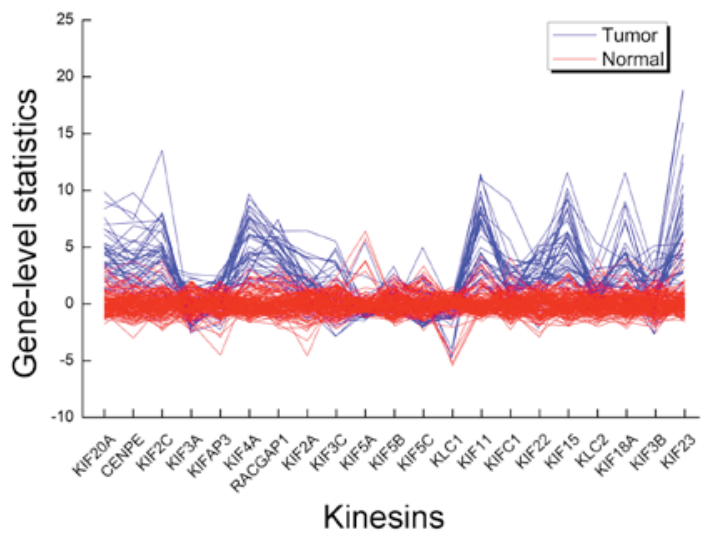

Figure 4. Gene level statistics in kinesins. Each line represents a sample. Blue, tumor; red, normal.

KIFs are a superfamily of microtubule-based motor proteins that exhibit diverse functions in the intracellular transportation of vesicles, organelles and chromosomes, the regulation of microtubule dynamics (21), and of molecular motors engaged in key cellular functions including cell division, mitosis and migration $(22,23)$. Previously, additional mitotic KIFs have been validated as drug targets for cancer drug development particularly for breast cancer, raising the possibility that the range of KIF-based drug targets may expand in the future (24). De et al (25) demonstrated that the over expression of KIF family member C3 (KIFC3), KIFC1, $K I F 1 A$, or $K I F 5 A$ to microtubules opposed the stabilizing effect of docetaxel that prevented cytokinesis and led to apoptosis. Similarly, the over expression of KIFC3, KIF5A, and $K I F 12$ were specific in mediating resistance to docetaxel and not vincristine or doxorubicin. This overexpression of KIFC3, $K I F 5 A$ and KIF12 correlated with specific taxane resistance in basal-like breast cancer; this ability was eliminated by a mutation of the adenosine triphosphate (ATP)-binding domain of a KIF (26). It had been identified that ANCCA (ATPase family, AAA nuclear coregulator cancer associated) is a key mediator of KIF family deregulation in breast 
Table III. Altered pathways with a percentage change $>80 \%$.

\begin{tabular}{|c|c|c|}
\hline Altered pathway & Amount & Percent $(\%)$ \\
\hline Kinesins & 39 & 92.86 \\
\hline Polo-like kinase mediated events & 39 & 92.86 \\
\hline Chromosome maintenance & 38 & 90.48 \\
\hline Meiotic recombination & 38 & 90.48 \\
\hline Deposition of new CENPA-containing nucleosomes at the centromere & 38 & 90.48 \\
\hline Nucleosome assembly & 38 & 90.48 \\
\hline $\mathrm{G}_{2} / \mathrm{M}$ DNA replication checkpoint & 38 & 90.48 \\
\hline Telomere maintenance & 37 & 88.10 \\
\hline Golgi cisternae pericentriolar stack reorganization & 37 & 88.10 \\
\hline $\begin{array}{l}\text { Nuclear factor-kB activation through Fas-associated death domain and receptor } \\
\text { interacting protein } 1 \text { pathway mediated by caspase- } 8 \text { and }-10\end{array}$ & 37 & 88.10 \\
\hline Phosphorylation of proteins involved in $\mathrm{G}_{1} / \mathrm{S}$ transition by active Cyclin $\mathrm{E} / \mathrm{Cdk} 2$ complexes & 37 & 88.10 \\
\hline Meiosis & 36 & 85.71 \\
\hline RNA polymerase I promoter clearance & 36 & 85.71 \\
\hline Amyloids & 36 & 85.71 \\
\hline Packaging of telomere ends & 36 & 85.71 \\
\hline RNA polymerase I promoter opening & 36 & 85.71 \\
\hline Cyclin A/B1 associated events during $\mathrm{G}_{2} / \mathrm{M}$ transition & 36 & 85.71 \\
\hline Leading strand synthesis & 36 & 85.71 \\
\hline Polymerase switching & 36 & 85.71 \\
\hline Polymerase switching on the C-strand of the telomere & 36 & 85.71 \\
\hline Phosphorylation of emi 1 & 36 & 85.71 \\
\hline Meiotic synapsis & 35 & 83.33 \\
\hline RNA polymerase I chain elongation & 35 & 83.33 \\
\hline $\mathrm{G}_{2} / \mathrm{M}$ checkpoints & 35 & 83.33 \\
\hline Activation of ATR in response to replication stress & 35 & 83.33 \\
\hline DNA strand elongation & 35 & 83.33 \\
\hline Activation of the pre-replicative complex & 35 & 83.33 \\
\hline Extension of telomeres & 35 & 83.33 \\
\hline Telomere C-strand (lagging strand) synthesis & 35 & 83.33 \\
\hline Resolution of AP sites via the multiple-nucleotide patch replacement pathway & 35 & 83.33 \\
\hline Zinc transporters & 35 & 83.33 \\
\hline Repair synthesis for gap-filling by DNA polymerase in TC-NER & 35 & 83.33 \\
\hline $\mathrm{E}_{2} \mathrm{~F}$-enabled inhibition of pre-replication complex formation & 35 & 83.33 \\
\hline ER quality control compartment & 35 & 83.33 \\
\hline Cyclin B2 mediated events & 35 & 83.33 \\
\hline Synthesis of DNA & 34 & 80.95 \\
\hline RNA polymerase I transcription & 34 & 80.95 \\
\hline Activation of $\mathrm{APC} / \mathrm{C}$ and $\mathrm{APC} / \mathrm{C}^{\mathrm{Cdc} 20}$ mediated degradation of mitotic proteins & 34 & 80.95 \\
\hline DNA damage bypass & 34 & 80.95 \\
\hline Translesion synthesis by Y family DNA polymerases bypasses lesions on DNA template & 34 & 80.95 \\
\hline $\mathrm{E}_{2} \mathrm{~F}$ mediated regulation of DNA replication & 34 & 80.95 \\
\hline $\mathrm{G}_{0}$ and early $\mathrm{G}_{1}$ & 34 & 80.95 \\
\hline Synthesis and interconversion of nucleotide di- and triphosphates & 34 & 80.95 \\
\hline $\mathrm{G}_{1} / \mathrm{S}$-specific transcription & 34 & 80.95 \\
\hline Phosphorylation of the APC/C & 34 & 80.95 \\
\hline Removal of the flap intermediate & 34 & 80.95 \\
\hline Chk1/Chk2(Cds1) mediated inactivation of cyclin B/Cdk1 complex & 34 & 80.95 \\
\hline
\end{tabular}

cancer and the crucial role of multiple KIFs in growth and survival of the tumor cells (27). Guerrero-Preston et al (28) suggested that differential promoter methylation of KIF1A in plasma was associated with breast cancer and DNA repair 
capacity. A negative correlation was identified between $K I F 2 A$ (KIF family member 2A) expression levels in breast cancer and the survival time of patients with breast cancer (29).

To additionally investigate functions of altered pathways, gene composition based on gene-level statistics were studied. A number of genes expressed deviated from the mean of the nRef, and these fluctuations may cause an alteration between breast cancer and normal controls. Among members of KIFs, KIF20A (KIF family member 20A) and KIF11 (KIS family member 11) were common genes between DEGs and pathway genes. For breast cancer patients, FOXM1 regulated KIF20A expression to modulate mitotic catastrophe caused by interferences in paclitaxel-mediated cell death and senescence (30). KIF11 represented an attractive anticancer target, and the inhibition of KIF11 caused mitotic arrest and apoptosis of multiple cancers, for example, breast cancer (31). Therefore, the KIF members and KIFs pathway had significant effects on breast cancer.

In the PLK mediated events pathway, PLK served a dominant role. PLK family members are known to be functionally involved in mitotic signaling, and in cytoskeletal reorganization in normal and malignant cells $(32,33)$. PLKs are also a family of conserved serine/threonine kinases involved in the regulation of cell cycle progression and in the activation of cyclin-dependent kinase/cyclin complexes during the $\mathrm{M}$-phase of the cell cycle through $\mathrm{G}_{2}$ and mitosis (34). Previous studies reported that PLK1 was a potential therapeutic option in combination with conventional chemotherapy for the management of patients with triple-negative breast cancer, and was overexpressed in tumors, indicating its involvement in carcinogenesis (35-37). The use of different PLK1 inhibitors has increased knowledge of mitotic regulation and allowed the present study to assess their ability to suppress tumor growth in vivo (38). The PLK2 and PLK3 acted in concert with cyclin-dependent kinase 1-cyclin B1 and aurora kinases to orchestrate a wide range of critical cell cycle events (39). As for other members of PLKs, there was evidence showing that PLK2 and PLK3 acted as tumor suppressors through their functions in the p53 signaling network, which guarded the cell against various stress signals $(40,41)$. It has been identified that there is a significant association between elevated $P L K 1$ and p53 mutation in women with breast cancer $(42,43)$. It has also been verified that $P L K 3$ is a novel independent prognostic marker in breast cancer, which alluded toward a role for PLK overexpression in disease progression (44). Therefore, it could be inferred that PLK mediated events correlate closely with breast cancer.

In conclusion, the iPAS method was suitable for identifying altered pathways (such as KIF- and PLK-mediated events), which may serve an important role in breast cancer progression and are potentially novel predictive and prognostic markers for breast cancer.

\section{References}

1. Müller A, Homey B, Soto H, Ge N, Catron D, Buchanan ME, McClanahan T, Murphy E, Yuan W, Wagner SN, et al: Involvement of chemokine receptors in breast cancer metastasis. Nature 410: 50-56, 2001.

2. DeSantis C, Ma J, Bryan L and Jemal A: Breast cancer statistics, 2013. CA Cancer J Clin 64: 52-62, 2014.
3. DeSantis C, Siegel R, Bandi P and Jemal A: Breast cancer statistics, 2011. CA Cancer J Clin 61: 408-418, 2011.

4. DiSipio T, Rye S, Newman B and Hayes S: Incidence of unilateral arm lymphoedema after breast cancer: A systematic review and meta-analysis. Lancet Oncol 14: 500-515, 2013.

5. Charif M, Lower EE, Kennedy D, Kumar H, Khan S, Radhakrishnan N and Zhang X: Abstract P3-05-16: The effect of HER-2/neu inhibition on prolonging clinical benefit with fulvestrant treatment for metastatic estrogen receptor positive breast cancer patients treated with trastuzumab. Cancer Res 75, 2015.

6. Mittendorf EA, Clifton GT, Holmes JP, Clive KS, Patil R, Benavides LC, Gates JD, Sears AK, Stojadinovic A, Ponniah S and Peoples GE: Clinical trial results of the HER-2/neu (E75) vaccine to prevent breast cancer recurrence in high-risk patients: From US Military Cancer Institute Clinical Trials Group Study I-01 and I-02. Cancer 118: 2594-2602, 2012.

7. Holder AM, Gonzalez-Angulo AM, Chen H, Akcakanat A Do KA, Fraser Symmans W, Pusztai L, Hortobagyi GN, Mills GB and Meric-Bernstam F: Increased stearoyl-CoA desaturase 1 expression is associated with shorter survival in breast cancer patients. Cancer Res 137: 319-327, 2013.

8. Glazko GV and Emmert-Streib F: Unite and conquer: Univariate and multivariate approaches for finding differentially expressed gene sets. Bioinformatics 25: 2348-2354, 2009.

9. Khatri P, Sirota M and Butte AJ: Ten years of pathway analysis: Current approaches and outstanding challenges. PLoS Comput Biol 8: e1002375, 2012.

10. Ahn T, Lee E, Huh N and Park T: Personalized identification of altered pathways in cancer using accumulated normal tissue data. Bioinformatics 30: i422-i429, 2014.

11. Chen DT, Nasir A, Culhane A, Venkataramu C, Fulp W, Rubio R, Wang T, Agrawal D, McCarthy SM, Gruidl M, et al: Proliferative genes dominate malignancy-risk gene signature in histologically-normal breast tissue. Breast Cancer Res Treat 119: 335-346, 2010.

12. Croft D, O'Kelly G, Wu G, Haw R, Gillespie M, Matthews L, Caudy M, Garapati P, Gopinath G, Jassal B, et al: Reactome: A database of reactions, pathways and biological processes. Nucleic Acids Res: 39 (Database issue): D691-D697, 2011.

13. Irizarry RA, Bolstad BM, Collin F, Cope LM, Hobbs B and Speed TP: Summaries of Affymetrix GeneChip probe level data. Nucleic Acids Res 31: e15, 2003.

14. Bolstad BM, Irizarry RA, Astrand M and Speed TP: A comparison of normalization methods for high density oligonucleotide array data based on variance and bias. Bioinformatics 19: 185-193, 2003.

15. Bolstad B: Affy: Built-in Processing Methods. 2013

16. Gehan EA: A Generalized Wilcoxon test for comparing arbitrarily singly-censored samples. Biometrika 52: 203-223, 1965.

17. Mohammadi A, Saraee MH and Salehi M: Identification of disease-causing genes using microarray data mining and Gene Ontology. BMC Med Genomics 4: 12, 2011.

18. Smyth GK: Linear models and empirical bayes methods for assessing differential expression in microarray experiments. Stat Appl Genet Mol Biol 3: 3, 2004.

19. Huang da W, Sherman BT and Lempicki RA: Systematic and integrative analysis of large gene lists using DAVID bioinformatics resources. Nat Protoc 4: 44-57, 2008.

20. Wang $X$ and Simon R: Microarray-based cancer prediction using single genes. BMC Bioinformatics 12: 391, 2011.

21. Rath O and Kozielski F: Kinesins and cancer. Nat Rev Cancer 12: 527-539, 2012.

22. Hirokawa N: Kinesin superfamily motor proteins and intracellular transport. Nat Rev Mol Cell Biol 10: 682-696, 2009.

23. Sharp DJ, Rogers GC and Scholey JM: Microtubule motors in mitosis. Nature 407: 41-47, 2000

24. Rath O and Kozielski F: Kinesins and cancer. Nat Rev Cancer 12: 527-539, 2012.

25. De S, Cipriano R, Jackson MW and Stark GR: Overexpression of kinesins mediates docetaxel resistance in breast cancer cells. Cancer Res 69: 8035-8042, 2009.

26. Tan MH, De S, Bebek G, Orloff MS, Wesolowski R, Downs-Kelly E, Budd GT, Stark GR and Eng C: Specific kinesin expression profiles associated with taxane resistance in basal-like breast cancer. Breast Cancer Res Treat 131: 849-858, 2012.

27. Zou JX, Duan Z, Wang J, Sokolov A, Xu J, Chen CZ, Li JJ and Chen HW: Kinesin family deregulation coordinated by bromodomain protein ANCCA and histone methyltransferase MLL for breast cancer cell growth, survival, and tamoxifen resistance. Mol Cancer Res 12: 539-549, 2014 
28. Guerrero-Preston R, Hadar T, Ostrow KL, Soudry E, Echenique M, Ili-Gangas C, Pérez G, Perez J, Brebi-Mieville P, Deschamps J, et al: Differential promoter methylation of kinesin family member 1a in plasma is associated with breast cancer and DNA repair capacity. Oncol Rep 32: 505-512, 2014.

29. Wang J, Ma S, Ma R, Qu X, Liu W, Lv C, Zhao S and Gong Y: KIF2A silencing inhibits the proliferation and migration of breast cancer cells and correlates with unfavorable prognosis in breast cancer. BMC Cancer 14: 461, 2014.

30. Khongkow P, Gomes A, Gong C, Man EP, Tsang JW, Zhao F, Monteiro LJ, Coombes RC, Medema RH, Khoo US and Lam EW: Paclitaxel targets FOXM1 to regulate KIF20A in mitotic catastrophe and breast cancer paclitaxel resistance. Oncogene 35 : 990-1002, 2016

31. Valensin S, Ghiron C, Lamanna C, Kremer A, Rossi M, Ferruzzi P, Nievo $\mathrm{M}$ and Bakker A: KIF11 inhibition for glioblastoma treatment: Reason to hope or a struggle with the brain? BMC Cancer 9: 196, 2009.

32. Sumara I, Vorlaufer E, Stukenberg PT, Kelm O, Redemann N, Nigg EA and Peters JM: The dissociation of cohesin from chromosomes in prophase is regulated by Polo-like kinase. Mol Cell 9: 515-525, 2002

33. Ha G, Kim H, Go H, Lee H, Seimiya H, Chung DH and Lee CW: Tankyrase-1 function at telomeres and during mitosis is regulated by Polo-like kinase-1-mediated phosphorylation. Cell Death Differ 19: 321-332, 2012.

34. von Schubert $C$ and Nigg EA: Polo-like kinases. Current Biol 23 : R225-R227, 2013.

35. Spänkuch-Schmitt B, Bereiter-Hahn J, Kaufmann M and Strebhardt K: Effect of RNA silencing of polo-like kinase-1 (PLK1) on apoptosis and spindle formation in human cancer cells. J Nat Cancer Inst 94: 1863-1877, 2002.

36. Maire V, Némati F, Richardson M, Vincent-Salomon A, Tesson B, Rigaill G, Gravier E, Marty-Prouvost B, De Koning L, Lang G, et al: Polo-like kinase 1: A potential therapeutic option in combination with conventional chemotherapy for the management of patients with triple-negative breast cancer. Cancer Res 3: 813-823, 2013
37. Wierer M, Verde G, Pisano P, Molina H, Font-Mateu J, Di Croce L and Beato M: PLK1 signaling in breast cancer cells cooperates with estrogen receptor-dependent gene transcription. Cell Rep 27: 2021-2032, 2013.

38. Strebhardt K and Ullrich A: Targeting polo-like kinase 1 for cancer therapy. Nat Rev Cancer 6: 321-330, 2006.

39. Strebhardt K: Multifaceted polo-like kinases: Drug targets and antitargets for cancer therapy. Nat Rev Drug Discov 9: 643-660, 2010.

40. Salvi M, Trashi E, Cozza G, Negro A, Hanson PI and Pinna LA: Tools to discriminate between targets of CK2 vs PLK2/PLK3 acidophilic kinases. Biotechniques 53, 2012.

41. Salvi M, Trashi E, Cozza G, Franchin C, Arrigoni G and Pinna L: Investigation on PLK2 and PLK3 substrate recognition. Biochim Biophys Acta 1824: 1366-1373, 2012.

42. King SI, Purdie CA, Bray SE, Quinlan PR, Jordan LB, Thompson AM and Meek DW: Immunohistochemical detection of Polo-like kinase-1 (PLK1) in primary breast cancer is associated with TP53 mutation and poor clinical outcom. Breast Cancer Res 14: R40, 2012.

43. Iyer RS, Nicol SM, Quinlan PR, Thompson AM, Meek DW and Fuller-Pace FV: The RNA helicase/transcriptional co-regulator, p68 (DDX5), stimulates expression of oncogenic protein kinase, Polo-like kinase-1 (PLK1), and is associated with elevated PLK1 levels in human breast cancers. Cell Cycle 13: 1413-1423, 2014.

44. Weichert W, Kristiansen G, Winzer KJ, Schmidt M, Gekeler V, Noske A, Müller BM, Niesporek S, Dietel M and Denkert C: Polo-like kinase isoforms in breast cancer: Expression patterns and prognostic implications. Virchows Archiv 446: 442-450, 2005. 